\title{
Does getting defensive get you anywhere? - Seasonally varying selection in pea aphids shapes a dynamic infection polymorphism with a protective bacterial endosymbiont
}

\author{
Drew Smith ${ }^{1}$, Michael O'Connor ${ }^{1}$, Brooke Deal ${ }^{1}$, Coleman Kotzer ${ }^{1}$, Amanda Lee ${ }^{1}$, Barrett \\ Wagner $^{1}$, Jonah Joffe ${ }^{2}$, Stephen Woloszynek ${ }^{1}$, Kerry Oliver ${ }^{3}$, and Jacob Russell ${ }^{1}$ \\ ${ }^{1}$ Drexel University \\ ${ }^{2}$ Affiliation not available \\ ${ }^{3}$ University of Georgia College of Agricultural and Environmental Sciences
}

July 7, 2020

\begin{abstract}
Facultative, heritable endosymbionts are found at intermediate prevalence within most insect species, playing frequent roles in their hosts' defense against environmental pressures. Focusing on Hamiltonella defensa, a common bacterial endosymbiont of aphids, we tested the hypothesis that such pressures impose seasonal balancing selection, shaping a widespread infection polymorphism. In our studied pea aphid (Acyrthosiphon pisum) population, Hamiltonella infection frequencies ranged from $23.2 \%$ to $68.1 \%$ across a six-month longitudinal survey. Rapid spikes and declines were consistent across fields, and we estimated that selection coefficients, for Hamiltonella-infected aphids, changed sign within this single season. Prior laboratory research suggested anti-parasitoid defense as the major Hamiltonella benefit, and costs under parasitoid absence. While a prior field study supported these forces as counter-weights in a regime of seasonal balancing selection, our present survey showed no significant relationship between parasitoid wasps and Hamiltonella. Field cage experiments provided some explanation: parasitoids drove $\sim 10 \%$ boosts to Hamiltonella frequencies that would be hard to detect under less controlled conditions. They also showed that Hamiltonella was not always costly under parasitoid exclusion, contradicting another long-held prediction. Instead, our longitudinal survey - and two overwintering studies - showed temperature to be the strongest predictor of Hamiltonella infection, matching some lab discoveries, and suggesting thermally sensitive costs and benefits, unrelated to parasitism, can shape this symbiont's prevalence. These results add to a growing body of evidence arguing for rapid, seasonal adaptation in multivoltine organisms. For many insects, such adaptation may be mediated through the diverse impacts of heritable symbionts on host phenotypes.
\end{abstract}

\section{Hosted file}

field cage paper v38b-no changes.docx available at https://authorea.com/users/337970/ articles/467666-does-getting-defensive-get-you-anywhere-seasonally-varying-selectionin-pea-aphids-shapes-a-dynamic-infection-polymorphism-with-a-protective-bacterialendosymbiont 
A. Stable Hamiltonella infection polymorphism

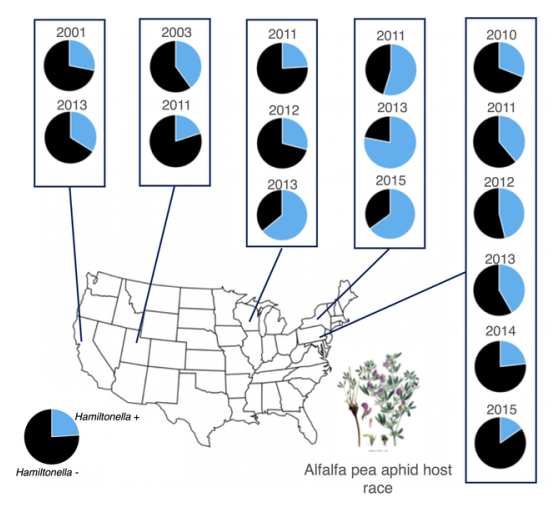

C. Experimental design and results
B. Hypothesized seasonal balancing selection

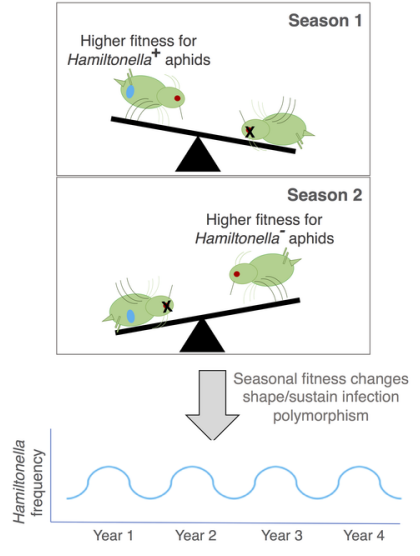

Testing the drivers of seasonal balancing selection

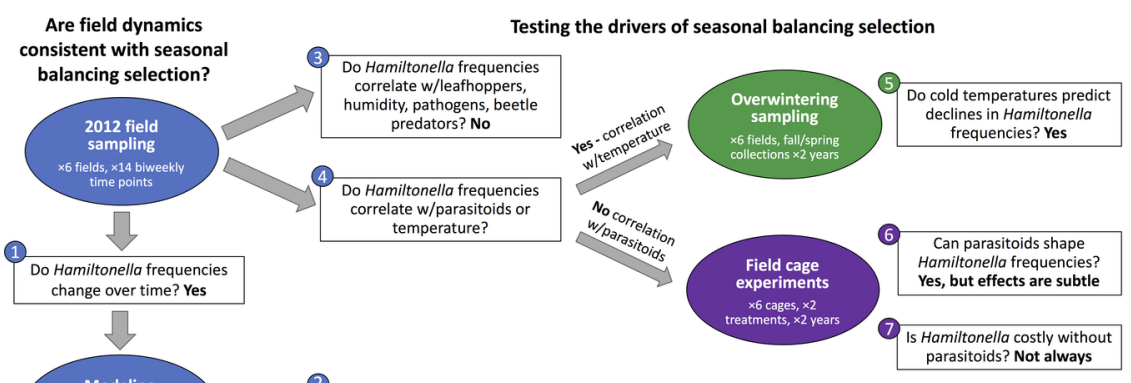


A.

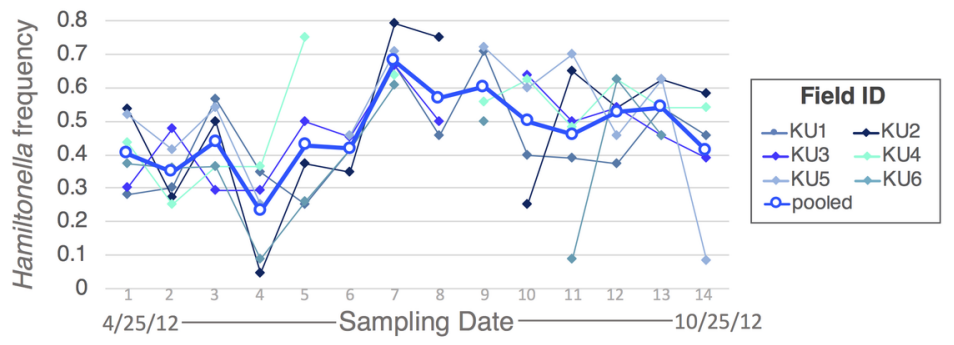

B.

Climatic variables

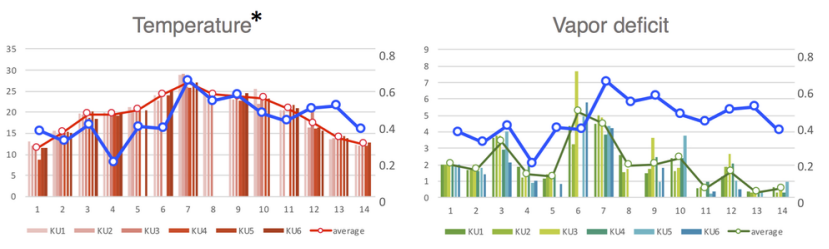

C.

Insect counts
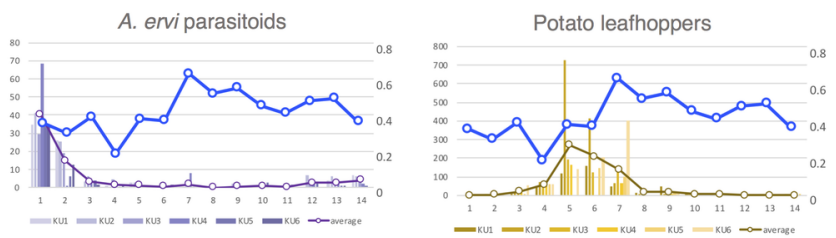

Coccinelid beetles
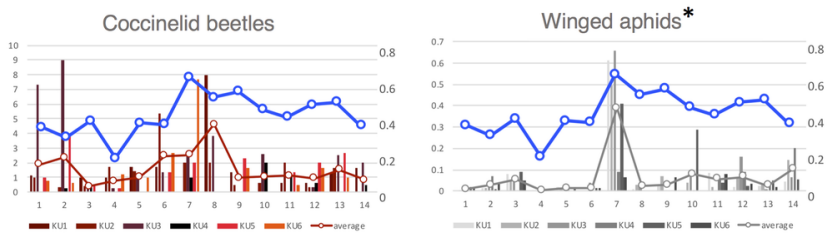

D.

Aphid fates after collection

Proportion A. ervi mummies
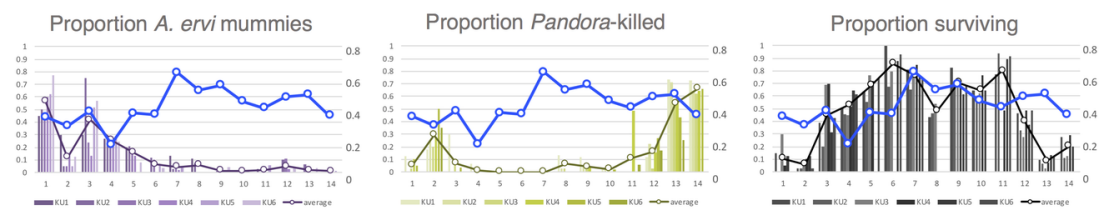


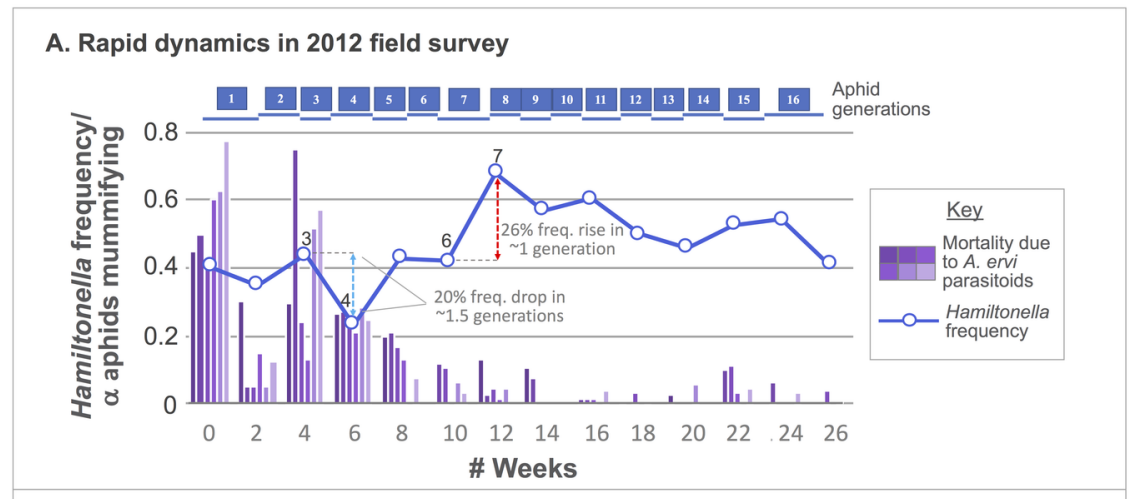

B. Changing selection coefficients (s) inferred from 2012 field data
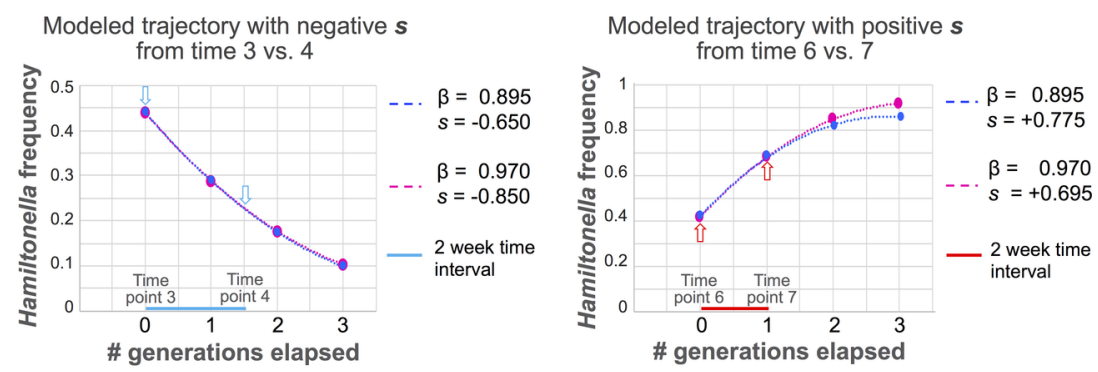

\section{Rapid dynamics in 2014 field cage experiment}

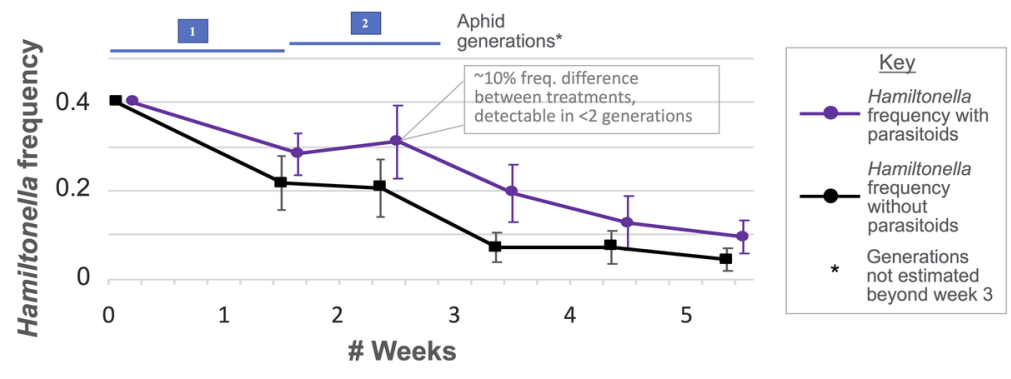

D. Rapid dynamics in 2008 lab cage experiment (Oliver et al. 2008)

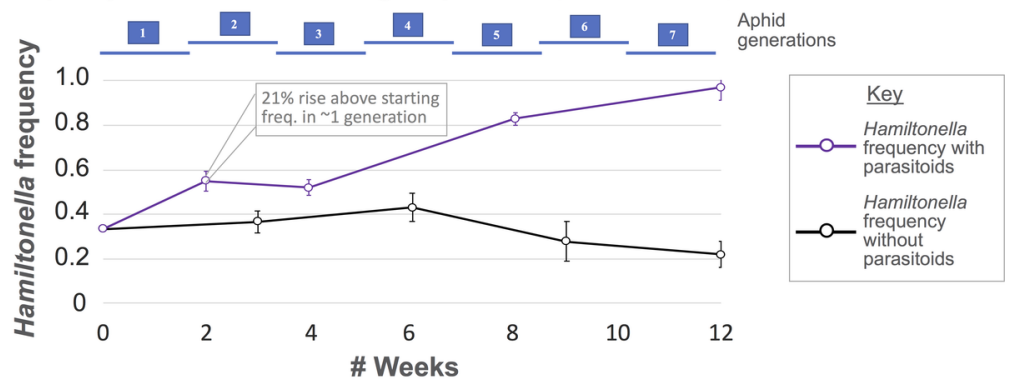


A.
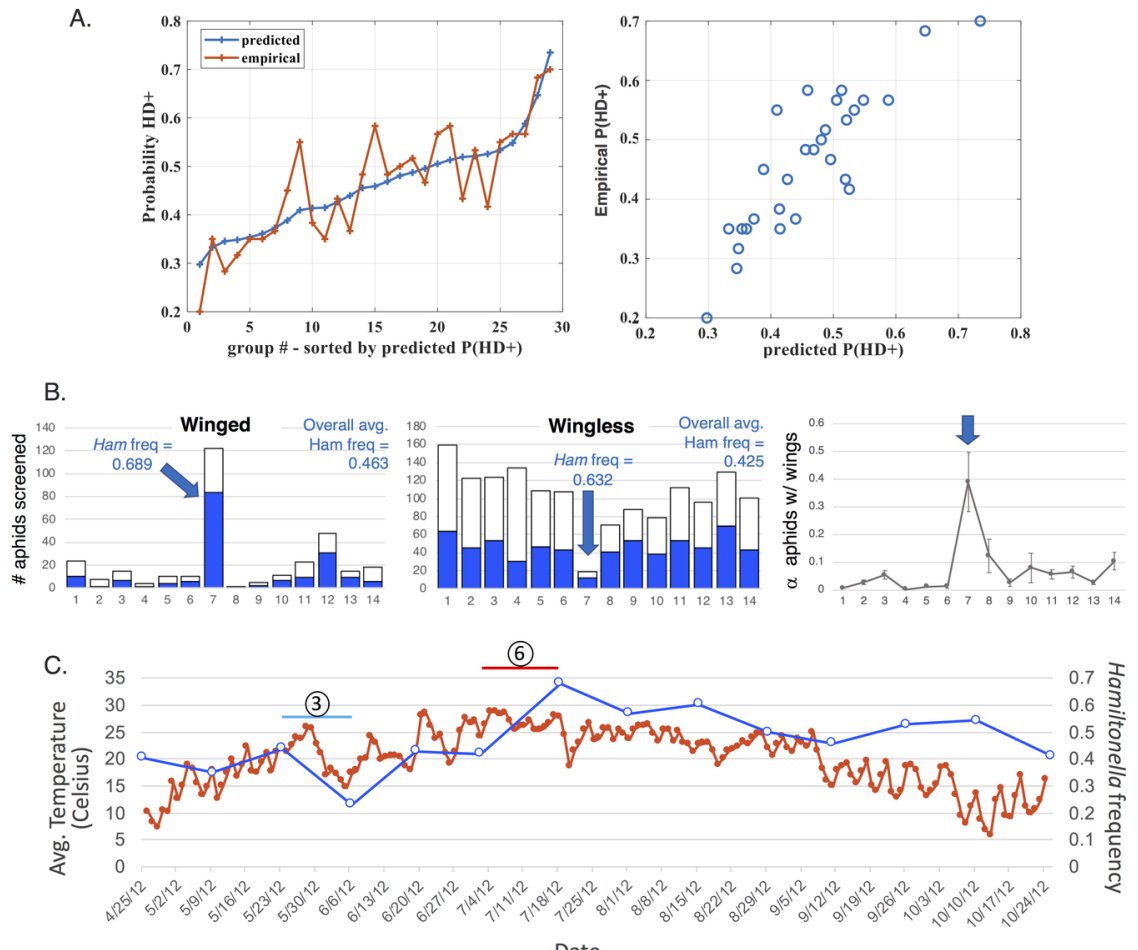

Date 


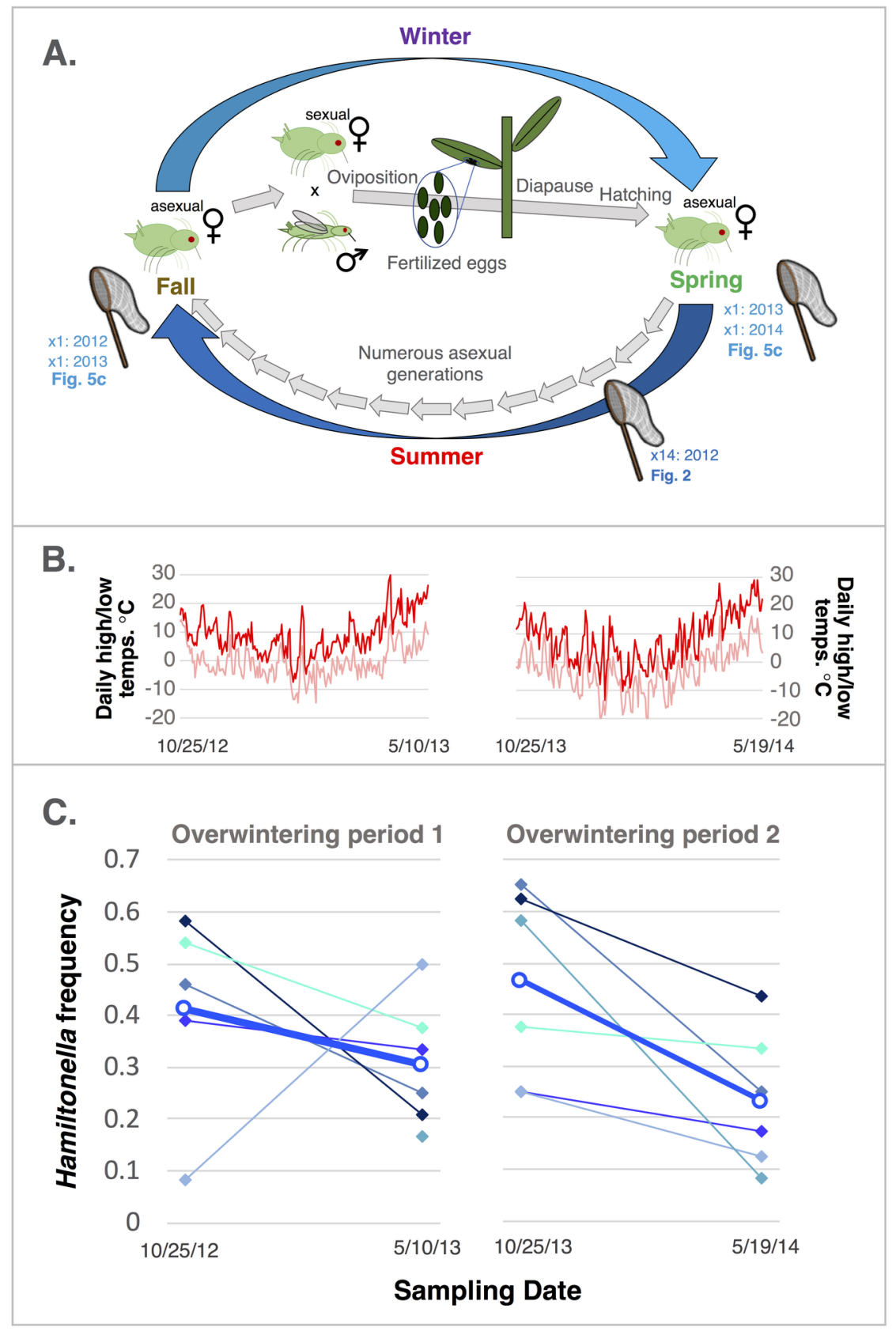




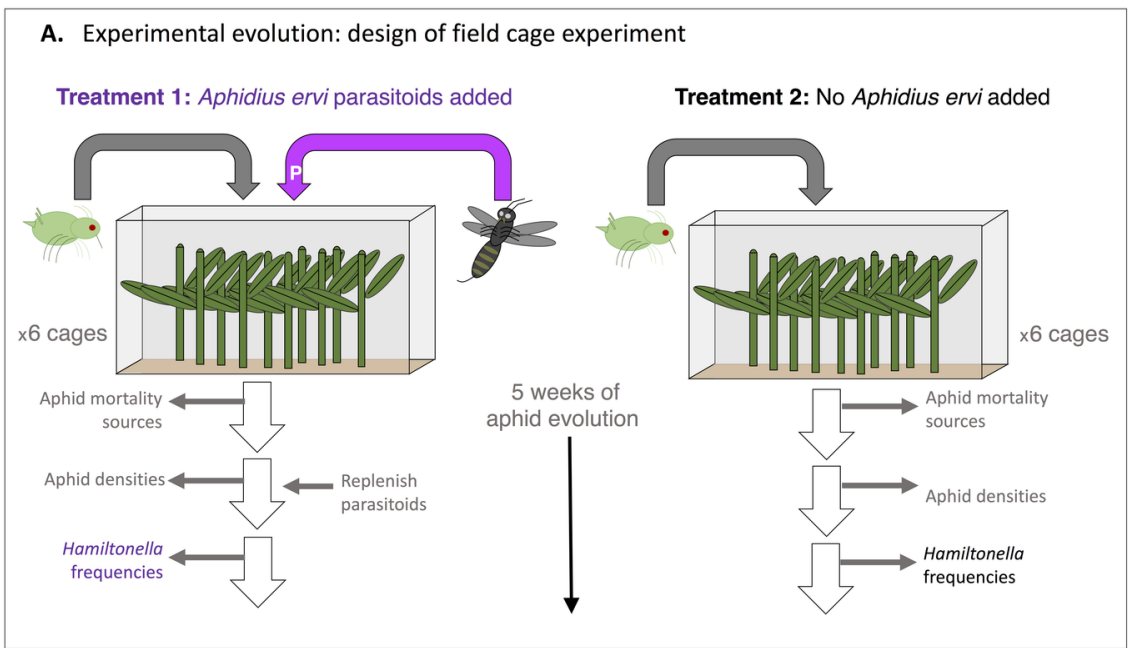

B. Predictions \& Results

2013 experiment

Hamiltonella does not defend experimental clone ZA17
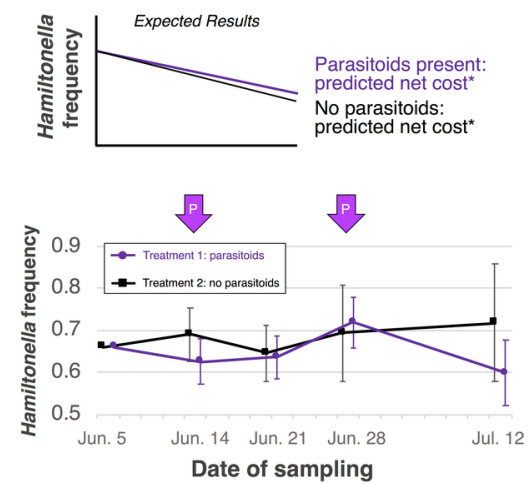

2014 experiment

Hamiltonella defends experimental clone A2E

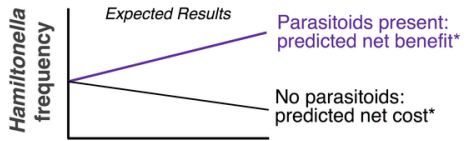

predicted net cost ${ }^{\star}$

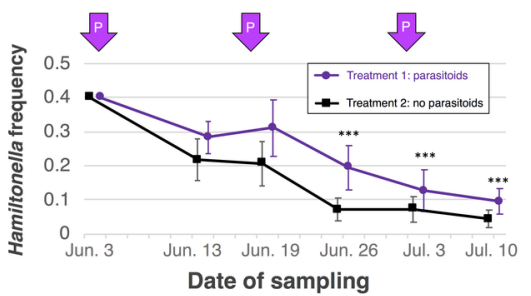

